Canadian

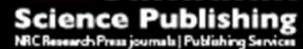

Canadian Journal of Microbiology Revue canadienne de de microbiologie

\title{
Might Dolutegravir Be Part of a Functional Cure for HIV?
}

\begin{tabular}{|r|l|}
\hline Journal: & Canadian Journal of Microbiology \\
\hline Manuscript ID & cjm-2015-0725.R1 \\
\hline Danuscript Type: & Review \\
\hline Complete List of Authors: & $\begin{array}{l}\text { Wainberg, Mark; Jewish General Hospital, , McGill University AIDS Centre, } \\
\text { Lady Davis } \\
\text { Han, Ying-Shan; McGill University AIDS Centre, Lady Davis for Medical } \\
\text { Research } \\
\text { Mesplede, Thibault; McGill University AIDS Centre, Lady Davis for Medical } \\
\text { Research }\end{array}$ \\
\hline Keyword: & \begin{tabular}{l} 
Dolutegravir, drug resistance, HIV cure \\
\hline
\end{tabular} \\
\hline
\end{tabular}

\section{SCHOLARONE ${ }^{\text {M }}$}

Manuscripts 
Might Dolutegravir Be Part of a Functional Cure for HIV?

Mark A. Wainberg ${ }^{1,2,3}$, Ying-Shan $\operatorname{Han}^{1}$ and Thibault Mesplède ${ }^{1}$

${ }^{1}$ McGill University AIDS Centre, Lady Davis for Medical Research, Jewish General Hospital, Montreal, Quebec, Canada; ${ }^{2}$ Division of Experimental Medicine and ${ }^{3}$ Department of

Microbiology and Immunology, Faculty of Medicine, McGill University, Montreal, Quebec, Canada.

*Corresponding author: Dr. Mark A. Wainberg (e-mail: mark.wainberg@mcgill.ca). 


\begin{abstract}
Antiretroviral therapy (ART) has greatly decreased HIV-related morbidity and mortality. However, HIV can establish viral reservoirs that evade both the immune system and ART. Dolutegravir (DTG) is a second-generation integrase strand transfer inhibitor (INSTI) related to the first generation INSTIs, raltegravir (RAL) and elvitegravir (EVG). DTG shows a higher genetic barrier to the development of HIV-1 resistance than RAL and EVG. More interestingly, clinical resistance mutations to DTG in treatment-naive patients have not been observed to date. This review summarizes recent studies on strategies toward to cure for HIV, resistance profiles of DTG and discusses how DTG might be possible in helping a functional cure for HIV.
\end{abstract}

Keywords:

Dolutegravir, drug resistance, HIV cure. 


\section{Introduction}

Antiretroviral therapy (ART) usually uses a combination of at least three antiretroviral (ARV) drugs that includes agents from more than one class. ART is effective in suppressing virus replication. In the past decades, the successful use of ART has greatly improved health and prolonged life in HIV-infected individuals, particularly when it is used in early stages of HIV infection. Currently, 29 antiretroviral drugs have been approved by the Food and Drug Administration (FDA) of the United States for use in the clinic. Based on their activities that target different stages of the HIV life cycle or host cellular factor, these drugs are classified into six different categories, including nucleoside reverse transcriptase inhibitors (NRTIs), nonnucleoside reverse transcriptase inhibitors (NNRTIs), protease inhibitors (PIs), fusion inhibitors, entry inhibitors and HIV integrase inhibitors.

Current ART is highly effective in suppressing virologic load in patients who are adherent to therapy. However, HIV can develop resistance to almost all classes of these antiretroviral drugs (Iyidogan and Anderson 2014). Moreover, HIV can establish latent HIV reservoir in memory $\mathrm{CD}^{+} \mathrm{T}$ cells, which are resistant to ART. The latent HIV viruses can reactivate and rebound within weeks in a majority of HIV-1-infected patients once ART is stopped (Solomon and Sax 2015). Thus, limitations of ART include a lifelong daily adherence to ART for individuals infected with HIV-1 and the risk of the emergence of virus mutants with multiclass resistance. Accordingly, there is a growing interest in approaches that will provide a cure or remission for HIV infection.

HIV mainly infects $\mathrm{CD}^{+} \mathrm{T}$ cells and macrophages in human blood and in other lymphoid organs, and the HIV genome is integrated into host cell genomes. The majority of the infected $\mathrm{CD}^{+} \mathrm{T}$ cells die quickly, but a small percentage can revert to a latent state in resting memory 
$\mathrm{CD}^{+} \mathrm{T}$ cells and become the virus reservoir. This virus reservoir is maintained in these individuals for a lifetime, even during ART. Ideally, a cure/eradication should mean complete elimination of HIV infected cells from the body. In contrast, a functional HIV cure might mean that patients do not have to take medications constantly, and can achieve undetectable viremia without either disease progression or HIV transmission. Current strategies for an HIV cure include starting ART very early before reservoir are fully established, eliminating residual virus replication by ART intensification, eliminating latently infected cells, keeping proviral DNA permanently silenced, enhancing HIV-specific immunity, and making cells resistant to HIV (Barouch and Deeks 2014; Dey and Berger 2015; Mylvaganam et al. 2015; Siliciano and Siliciano 2014).

Integrase strand transfer inhibitors (INSTIs) are the latest class of antiretroviral drugs. INSTIs block the integration of HIV viral DNA into host chromosomal DNA. Currently, three INSTIs were approved by FDA and these are raltegravir (RAL) and elvitegravir (EVG), i.e. the first generation INSTIs, and dolutegravir (DTG), a second generation INSTI. Although RAL and EVG are highly effective in treatment of patients with HIV, HIV resistance and cross resistance to both RAL and EVG can emerge. However, DTG has shown a superior barrier to HIV resistance compared to RAL and EVG. Importantly it has not been observed that HIV can develop resistance mutations against DTG when the drug is used in first line therapy. This unique resistance profile might make DTG useful in thinking about a functional cure for HIV. In this review, we summarize recent studies on potential approaches towards a functional HIV cure, the resistance profile of DTG, and discuss our understandings on how DTG might play a role in helping to achieve a functional HIV cure. 


\section{Current Potential HIV Cure Strategies}

A few years ago, there was debate as to whether or not HIV is curable, with many arguing against this possibility (Purcell et al. 2013). Now, however, there is increasing evidence and confidence that at least a functional cure for HIV might be achieved.Monitoring of the latest studies on HIV cure/remission has revealed a number of strategies that are being pursued (Barouch and Deeks 2014; Dey and Berger 2015; Martin and Siliciano 2015; Pace and Frater 2014; Thornhill et al. 2015).

Most important is the case of the Berlin Patient, the first person who has been cured of HIV (Allers et al. 2011; Hutter et al. 2009). This patient had lived with HIV for 11 years while under therapy with ART and, in 2007, developed acute myeloid leukemia. Then he received a stem cell transplant from a donor who was homozygous for a 32-bp deletion in the CCR5 allele, which provides resistance against HIV-1 infection. The patient remains free of any sign of HIV rebound and disease progression over the past 8 years after transplantation and discontinuation of ART. This case provides a proof of principle that a HIV cure might be possible. However, this approach is not practical and it remains necessary to find one that is more satisfying and broadly applicable.

Another potential approach for an HIV cure is a "shock and kill" strategy. This approach first uses latency-reversing agents to induce latently infected cells to produce virus ("shock") and then strive to clear these virus-producing cells ("kill") through an enhancement of host antiviral immunity (Barouch and Deeks 2014). The latency-reversing agents mainly include histone deacetylase (HDAC) inhibitors, methylation inhibitors, and cytokine (e.g. interleukin-7). Recently, it was reported that ingenol-3-angelate (PEP005), a FDA approved anticancer drug, can effectively reactivate latent HIV in vitro and ex vivo with relatively low cellular toxicity 
(Jiang et al. 2015). These compounds are potential candidates for advancing HIV eradication. The immunologic strategies for killing the virus-producing cells include therapeutic vaccines, monoclonal antibodies, and immune checkpoint inhibitors (Barouch and Deeks 2014; Katlama et al. 2013). This strategy is currently under investigation in clinical trials.

For example, a trial in the UK will evaluate the combination of vorinostat, a HDAC inhibitor, and vaccination (Thornhill et al. 2015). To attack the reservoir, it is important to locate the sites at which the latently infected cells reside. Current approaches mainly focus on $\mathrm{CD}^{+} \mathrm{T}$-cells in blood. But other cell types or tissues may also harbour latent virus, including monocytes, macrophages, and T-memory stem cells. A large proportion of latently infected cells may also reside in tissues outside of blood, including lymphoid tissue, the central nervous system (CNS), spleen and lymph nodes. New assays to identify and measure latent reservoirs of HIV-infected cells need to be validated (Bruner et al. 2015).

Nowadays, ART is widely recognized as being able to inhibit HIV replication and reduce viral load in HIV-infected patients, but the effect of ART drugs on viral latency and immune activation is still poorly understood (Hladik 2015). It has been reported that early ART during acute HIV infection reduced the size of reservoir (Ananworanich et al. 2015; Ananworanich et al. 2012; Archin et al. 2012) and it is possible that very early ART may be curative for HIV. A French girl was infected with HIV when she was born in 1996. She received a prophylactic treatment for six weeks from the day she was born. She was switched to ART from three months of age until about the age of five years six months when ART was stopped. She returned to the clinic after discontinuation of ART for a full year. It was found that she remained undetectable in terms of viral load in her blood at that time and no treatment was given. She has now been in remission from infection without ART for 12 years (Saez-Cirion 2015). However, a strong 
limitation is that virtually all HIV-infected patients are only diagnosed months or years after infection has taken place, i.e. long after the reservoirs have been seeded with HIV.

Early ART might also be able to prevent some of the immunologic damage caused by HIV, even it fails to block the establishment of the reservoir (Barouch and Deeks 2014). A cohort of 14 HIV-positive patients in France were treated with ART for at least three years, at which time ART was stopped (the VISCONTI cohort). One patient out of the 14 adults did not show any sign of viral rebound after discontinuation of ART for 13 years (Autran 2015; Saez-Cirion et al. 2013). A recent study has shown that early ART initiated during primary infection permits posttreatment control of HIV infection by limiting the size of the latent reservoir, which, if small enough at treatment termination, may allow the adaptive immune response to prevent viral rebound and control infection (Conway and Perelson 2015). Some clinical studies have shown that early ART not only inhibits HIV-1 replication, but also limits the size of viral reservoirs. Continued ART with a reduction in HIV-1 reservoirs over time may facilitate HIV-1 eradication strategies (Hocqueloux et al. 2013; Jain et al. 2013; Li and Gandhi 2014; Luzuriaga et al. 2014).

It was recently reported that a Tat inhibitor didehydro-cortistatin A (dCA) reduces residual levels of viral transcription in several models of HIV latency and establishes a nearly permanent state of latency. In cell culture experiments, treatment with dCA induces inactivation of viral transcription, even after its removal. This study suggested that a Tat inhibitor in combination with current ART regimens may contribute to a functional HIV-1 cure by reducing low-level viremia and permanently silencing latent reservoirs (Mousseau et al. 2015).

Recent novel strategies include neutralizing HIV-specific monoclonal antibodies (Barouch et al. 2013), ART intensification (DaFonseca et al. 2015), and gene editing (Allers and Schneider 2015; Khalili et al. 2015). Since each strategy has some limitations, it is most likely that a 
functional cure may require a combination of various approaches. The development of novel strategies will be essential to attain this goal.

\section{The Unique HIV Drug Resistance Profile of DTG}

RAL and EVG are effective in inhibiting HIV replication and reducing viral load in HIVinfected individuals. However, resistance to RAL and EVG can emerge rapidly both in vitro and in patients (Geretti et al. 2012). Three major mutational pathways have been described as conferring resistant to RAL and begin with mutations at positions Y143, Q148, or N155 in integrase. The major primary mutations that initiate resistant to EVG include T66I, E92Q, $\mathrm{N} 155 \mathrm{H}$ and $\mathrm{Q} 148 \mathrm{H} / \mathrm{K} / \mathrm{R}$. Thus cross resistance exists between RAL and EVG in regard to mutations at position 155 and 148. In contrast, DTG has a higher barrier to HIV drug resistance compared to RAL and EVG (Llibre et al. 2015; Seki et al. 2015; Wainberg and Han 2015; White et al. 2014). The major mutations that confer resistance to RAL, EVG and DTG are summarized in Table 1.

It is important to point out that DTG can be distinguished from both RAL and EVG in the sense that very limited cross-resistance exists among these compounds (Mesplede and Wainberg 2015; Wainberg and Han 2015). Some mutations in integrase that are potentially involved in resistance to DTG were reported at positions at F121, S153, G118, E138, and R263 in vitro and in vivo (Kobayashi et al. 2011; Quashie et al. 2013b). R263K was initially reported as the most common substitution identified in cell culture selections with DTG and was shown to confer only moderate resistance to DTG (fold change, $\mathrm{FC}=2.3$-fold) (Quashie et al. 2012). Biochemical studies also showed that R263K decreased strand transfer activity and viral replication capacity. 
Later, it was reported that R263K was present in several treatment-experienced, INSTI-naïve patients in the SAILING clinical trial (Cahn et al. 2013).

In the development of HIV resistance to drugs, it is common that a primary mutation confers a low level of resistance while reducing replication capacity and that the subsequent occurrence of a combination of secondary mutations increase levels of drug resistance while restoring viral replication fitness. In cell culture selections with DTG, R263K was often observed together with H51Y. While the latter had no effects on resistance to DTG, the addition of H51Y to R263K increased resistance to DTG to $\sim 8$-fold, while dramatically decreasing viral replication capacity by $\sim 90 \%$ and enzyme strand transfer activity by $\sim 80 \%$ (Mesplede et al. 2013). Other studies have shown that R263K in combination with M50I, G118R, H51Y, E138K, T66I, N155H or M184I/V slightly increased resistance to DTG, but did not restore viral replication capacity (Anstett et al. 2015; Liang et al. 2015; Quashie et al. 2013a; Quashie et al. 2015; Wares et al. 2014). Interestingly, no additional compensatory mutations have been identified for DTG, even in cell culture selection experiments conducted over more than four years (Wainberg and Han 2015).

It is generally believed that the higher genetic barrier of DTG to resistance of HIV-1 is due to its slow dissociation rate from integrase-DNA complexes in comparison to RAL and EVG (Geretti et al. 2012; Hightower et al. 2011; Osman et al. 2015; Wainberg and Han 2015). Some studies have shown that DTG has an extended linker which allows its difluorophenyl group to enter farther into the pocket within the integrase active site than other INSTIs and that DTG has the ability to adjust its structure and conformation in response to structural changes within the active sites of RAL- and EVG-resistant integrases, compared to RAL and EVG (Fig. 1) (Hare et al. 2011; Quashie et al. 2013a). A recent study has shown that DTG exhibits anti-HIV activity in tissue culture more effectively than that of other INSTIs after drug washout, perhaps due to its 
prolonged residency time within integrase (Osman et al. 2015). This result was observed even if the $\mathrm{R} 263 \mathrm{~K}$ mutation was present.

So far, virological failure has not yet been reported in treatment-naive individuals receiving DTG-containing regimens in the clinic. This may be partially due to the fact that the R263K substitution, that confer low resistance to DTG, can impair the ability of HIV to develop additional resistance substitutions (Wainberg et al. 2014). A recent study measured the genetic and amino-acid diversity of Env/gp160 from two HIV-1 primary isolates that were grown in the presence of increasing concentrations of DTG or RAL over the course of 38-55 weeks. It was found that treatment with DTG led to less HIV-1 genetic and amino-acid diversity over time, as compared to treatment with RAL or the absence of drug (Mesplede et al. 2015). These results may also help to explain the absence of emergent resistance mutations for any drug in treatmentnaive individuals treated with DTG, as a result of more limited HIV evolution in patients treated with the latter drug.

\section{How Might DTG Be Useful in a Functional Cure for HIV?}

Until now, the use of DTG in first-line therapy has not yielded any resistance mutations in HIV-patients in the clinic. Data from tissue culture selection with DTG revealed that the development of an initial HIV resistance mutation for DTG was associated with greatly reduced replication capacity. Due to its efficacy and high barrier to resistance, DTG is becoming one of the preferred choices for HIV therapy in both treatment-naïve and treatment-experienced patients (Cruciani and Malena 2015). Since DTG has a truly unique resistance profile, in which the resistance mutations that are selected by the drug result in a severe impairment in replication fitness, concomitant with a greatly diminished capacity to generate additional mutations, we 
believe that DTG might be able to be used as part of a strategy aimed at attaining a functional HIV cure if not complete HIV eradication.

For one thing, if DTG is imperious to the occurrence of resistance in first-line therapy, then it might be possible to use it as monotherapy in treatment-naive patients. In this case, even if the R263K mutation is selected, the viruses would not be able to grow well, due to impaired replication capacity. Following interruption of DTG-containing treatment, viruses might begin to replicate from the latent reservoirs, as observed in other ART treatment interruption trials. However, re-initiation of DTG monotherapy might then convert these viruses into DTG-resistant attenuated forms. Logically, a number of cycles of DTG treatment interruption followed by reinitiation of DTG monotherapy could conceivably convert all the HIV in the body, even that within latent reservoirs, as they emerge out of latency, to a replication impaired form. As viral reservoirs decay over a number of cycles, the reduced HIV-1 reservoirs would not be able to rebound once interruption of ART takes place (Fig. 2). If this is true, such an approach might lead to a functional cure of HIV disease if all residual viruses were significantly impaired in viral replication and thereby reduced in numbers and if further compensatory mutations were unable to develop (Mesplede and Wainberg 2014; Wainberg and Han 2015). Recent studies have shown that early initiation of antiretroviral therapy can greatly reduce the size of the HIV reservoir and help to achieve optimal immune reconstitution (Buzon et al. 2014; Conway and Perelson 2015; Hocqueloux et al. 2013).

Obviously, a cure approach will depend on a number of factors, such as the size of the latent reservoir, the rate of reactivation of latent viruses, and the strength of the host immune response (Conway and Perelson 2015). At present, in the absence of sufficient clinical data, it is difficult to estimate how many cycles of interruption and resumption of DTG therapy might be needed to 
achieve a possible functional cure or what the duration of therapy of each cycle should be. However, strategies aimed at reducing the latent reservoir size and/or boosting host immune responses, combined with the DTG interruption approach, may enhance the probability of a possible cure. In the Visconti study, some patients remained aviremic while being off therapy for a median of 89 months, after having received a median of 36 months of ART (Thornhill et al. 2015). It might therefore be reasonably predicted that DTG interruption and resumption over several cycles might also be able to contribute to a purging of the numbers of archived wild-type viruses, especially if combined with other approaches.

A proof of concept study should ideally first be conducted in animal models, such as rhesus macaques that are infected by simian immunodeficiency virus (SIV) or humanized mice that are infected by HIV. Of course, until such animal studies are complete, we will be without data to prove that this approach will actually lead to the replacement of wild-type viruses in the reservoirs by DTG-resistant defective viruses. Additionally, the rate at which defective viruses may replace WT viruses within the reservoirs is unknown, and this process may take decades before it yields a clinically significant decrease in the size of the WT reservoirs. In this regard, it is important to note that viral reservoirs become established early during the course of infection, as has been shown in rhesus monkeys infected with SIV (Whitney et al. 2014). The latter is thus an excellent model to study the ability of defective viruses to replace replication-competent WT viruses.

In addition, the extent to which the viral reservoirs must be diminished to attain long-term remission remains unknown. Therefore, even if defective viruses replace $90 \%$ of the WT viruses in reservoirs, this may not suffice to allow immunological control of residual viral replication to occur following treatment interruption. This issue is of concern for all current cure strategies and 
these strategies will need to be addressed in animal models prior to being tested in individuals living with HIV.

Some clinical studies with DTG monotherapy, such as dose-ranging studies, have been done in the past. More clinical studies are needed in which patients would first be suppressed with DTG plus two other drugs and then maintained on DTG monotherapy. Resistance mutations to DTG in both the RNA of patient plasma samples and the DNA of patient PBMCs would need to be intensively monitored by ultrasensitive sequencing methods. If the results show an absence of resistance to DTG, this would help to validate the above hypothesis.

It has been argued that it might only be a matter of time until the development of compensatory mutations that are associated with DTG. Data from cell culture selection experiments have shown that no further resistance mutations have been observed after more than four years of DTG selective pressure (Wainberg and Han 2015). Moreover, it is possible that DTG will be the drug of choice in a variety of prevention strategies, such as Treatment as Prevention. Should this turn out to be the case, there might be profound implications both for the prevention of HIV transmission and HIV cure and/or remission.

\section{Conclusions and Perspectives}

The past several years have seen important advances in research toward a HIV cure. Increasing evidence has suggested that HIV-1 might be eradicated or that a functional cure might be possible. However, more detailed understanding of the biology of the latent viral reservoir and the relevant virus-specific immune response is needed, in addition to improved latency assays to quantitate the viral reservoir (Bruner et al. 2015). Reliable animal models should be developed to evaluate concepts and strategies. 
Early ART initiation and treatment intensification with integrase inhibitors may significantly improve immunity in HIV-infected subjects (DaFonseca et al. 2015) as it seems that the establishment and maintenance of viral reservoirs are at least, in part, affected by the immune system. Immunotherapeutic approaches are likely to play an increasing role in establishment of HIV-1 remission. Although a number of reports shows that it might be possible to cure HIV, it is unlikely that only one approach will lead to this goal and multiple promising strategies for a HIV cure should be explored. More studies on the effects of DTG on viral latency, immune activation and HIV evolution are needed (Barouch and Deeks 2014; Simonetti and Kearney 2015), as is a more detailed understandings of the host determinants that govern latency and reservoir size during ART therapy (Abdel-Mohsen et al. 2015).

\section{Acknowledgement}

Work performed in our laboratory has been funded by the Canadian Institutes for Health Research (CIHR). 


\section{References}

Abdel-Mohsen, M., Wang, C., Strain, M.C., Lada, S.M., Deng, X., Cockerham, L.R., Pilcher, C.D., Hecht, F.M., Liegler, T., Richman, D.D., Deeks, S.G., and Pillai, S.K. 2015. Select host restriction factors are associated with HIV persistence during antiretroviral therapy. AIDS (London, England) 29(4): 411-420. doi: $10.1097 /$ qad. 0000000000000572 .

Allers, K., Hutter, G., Hofmann, J., Loddenkemper, C., Rieger, K., Thiel, E., and Schneider, T. 2011.

Evidence for the cure of HIV infection by CCR5Delta32/Delta32 stem cell transplantation. Blood 117(10): 2791-2799. doi: 10.1182/blood-2010-09-309591.

Allers, K., and Schneider, T. 2015. CCR5Delta32 mutation and HIV infection: basis for curative HIV therapy. Current opinion in virology 14: 24-29. doi: 10.1016/j.coviro.2015.06.007.

Ananworanich, J., Dube, K., and Chomont, N. 2015. How does the timing of antiretroviral therapy initiation in acute infection affect HIV reservoirs? Current opinion in HIV and AIDS 10(1): 18-28. doi: 10.1097/coh.0000000000000122.

Ananworanich, J., Schuetz, A., Vandergeeten, C., Sereti, I., de Souza, M., Rerknimitr, R., Dewar, R., Marovich, M., van Griensven, F., Sekaly, R., Pinyakorn, S., Phanuphak, N., Trichavaroj, R., Rutvisuttinunt, W., Chomchey, N., Paris, R., Peel, S., Valcour, V., Maldarelli, F., Chomont, N., Michael, N., Phanuphak, P., and Kim, J.H. 2012. Impact of multi-targeted antiretroviral treatment on gut T cell depletion and HIV reservoir seeding during acute HIV infection. PloS one 7(3): e33948. doi: 10.1371/journal.pone.0033948. Anstett, K., Fusco, R., Cutillas, V., Mesplede, T., and Wainberg, M.A. 2015. Dolutegravir-Selected HIV-1 Containing the N155H and R263K Resistance Substitutions Does Not Acquire Additional Compensatory Mutations under Drug Pressure That Lead to Higher-Level Resistance and Increased Replicative Capacity. Journal of virology 89(20): 10482-10488. doi: 10.1128/jvi.01725-15. 
Archin, N.M., Vaidya, N.K., Kuruc, J.D., Liberty, A.L., Wiegand, A., Kearney, M.F., Cohen, M.S., Coffin, J.M., Bosch, R.J., Gay, C.L., Eron, J.J., Margolis, D.M., and Perelson, A.S. 2012. Immediate antiviral therapy appears to restrict resting CD4+ cell HIV-1 infection without accelerating the decay of latent infection. Proceedings of the National Academy of Sciences of the United States of America 109(24): 9523-9528. doi: 10.1073/pnas.1120248109.

Autran, B. 2015. Robust HIV-specific T cells in post-treatment controllers from the VISCONTI cohort. 8th IAS Conf. on HIV Pathogenesis, Treatment and Prevention 19-22 July 2015, Vancouver: TUPEA093. Barouch, D.H., and Deeks, S.G. 2014. Immunologic strategies for HIV-1 remission and eradication. Science 345(6193): 169-174. doi: 10.1126/science.1255512.

Barouch, D.H., Whitney, J.B., Moldt, B., Klein, F., Oliveira, T.Y., Liu, J., Stephenson, K.E., Chang, H.W., Shekhar, K., Gupta, S., Nkolola, J.P., Seaman, M.S., Smith, K.M., Borducchi, E.N., Cabral, C., Smith, J.Y., Blackmore, S., Sanisetty, S., Perry, J.R., Beck, M., Lewis, M.G., Rinaldi, W., Chakraborty, A.K., Poignard, P., Nussenzweig, M.C., and Burton, D.R. 2013. Therapeutic efficacy of potent neutralizing HIV-1-specific monoclonal antibodies in SHIV-infected rhesus monkeys. Nature 503(7475): 224-228. doi: 10.1038/nature12744.

Bruner, K.M., Hosmane, N.N., and Siliciano, R.F. 2015. Towards an HIV-1 cure: measuring the latent reservoir. Trends in microbiology 23(4): 192-203. doi: 10.1016/j.tim.2015.01.013.

Buzon, M.J., Martin-Gayo, E., Pereyra, F., Ouyang, Z., Sun, H., Li, J.Z., Piovoso, M., Shaw, A., Dalmau, J., Zangger, N., Martinez-Picado, J., Zurakowski, R., Yu, X.G., Telenti, A., Walker, B.D., Rosenberg, E.S., and Lichterfeld, M. 2014. Long-term antiretroviral treatment initiated at primary HIV-1 infection affects the size, composition, and decay kinetics of the reservoir of HIV-1-infected CD4 T cells. Journal of virology 88(17): 10056-10065. doi: 10.1128/jvi.01046-14.

Cahn, P., Pozniak, A.L., Mingrone, H., Shuldyakov, A., Brites, C., Andrade-Villanueva, J.F., Richmond, G., Buendia, C.B., Fourie, J., Ramgopal, M., Hagins, D., Felizarta, F., Madruga, J., Reuter, T., Newman, T., 
Small, C.B., Lombaard, J., Grinsztejn, B., Dorey, D., Underwood, M., Griffith, S., and Min, S. 2013.

Dolutegravir versus raltegravir in antiretroviral-experienced, integrase-inhibitor-naive adults with HIV: week 48 results from the randomised, double-blind, non-inferiority SAILING study. Lancet 382(9893): 700-708. doi: 10.1016/s0140-6736(13)61221-0.

Conway, J.M., and Perelson, A.S. 2015. Post-treatment control of HIV infection. Proceedings of the National Academy of Sciences of the United States of America 112(17): 5467-5472. doi:

10.1073/pnas.1419162112.

Cruciani, M., and Malena, M. 2015. Combination dolutegravir-abacavir-lamivudine in the management of HIV/AIDS: clinical utility and patient considerations. Patient preference and adherence 9: 299-310. doi: $10.2147 /$ ppa.s65199.

DaFonseca, S., Niessl, J., Pouvreau, S., Wacleche, V.S., Gosselin, A., Cleret-Buhot, A., Bernard, N., Tremblay, C., Jenabian, M.A., Routy, J.P., and Ancuta, P. 2015. Impaired Th17 polarization of phenotypically naive CD4(+) T-cells during chronic HIV-1 infection and potential restoration with early ART. Retrovirology 12: 38. doi: 10.1186/s12977-015-0164-6.

Dey, B., and Berger, E.A. 2015. Towards an HIV cure based on targeted killing of infected cells: different approaches against acute versus chronic infection. Current opinion in HIV and AIDS 10(3): 207-213. doi: 10.1097/coh.0000000000000151.

Geretti, A.M., Armenia, D., and Ceccherini-Silberstein, F. 2012. Emerging patterns and implications of HIV-1 integrase inhibitor resistance. Current opinion in infectious diseases 25(6): 677-686. doi: 10.1097/QCO.0b013e32835a1de7.

Hare, S., Smith, S.J., Metifiot, M., Jaxa-Chamiec, A., Pommier, Y., Hughes, S.H., and Cherepanov, P. 2011. Structural and functional analyses of the second-generation integrase strand transfer inhibitor dolutegravir (S/GSK1349572). Molecular pharmacology 80(4): 565-572. doi: 10.1124/mol.111.073189. 
Hightower, K.E., Wang, R., Deanda, F., Johns, B.A., Weaver, K., Shen, Y., Tomberlin, G.H., Carter, H.L., 3rd, Broderick, T., Sigethy, S., Seki, T., Kobayashi, M., and Underwood, M.R. 2011. Dolutegravir (S/GSK1349572) exhibits significantly slower dissociation than raltegravir and elvitegravir from wild-type and integrase inhibitor-resistant HIV-1 integrase-DNA complexes. Antimicrobial agents and chemotherapy 55(10): 4552-4559. doi: 10.1128/aac.00157-11.

Hladik, F. 2015. A new hypothesis on HIV cure. F1000Research 4: 77. doi:

10.12688/f1000research.4529.1.

Hocqueloux, L., Avettand-Fenoel, V., Jacquot, S., Prazuck, T., Legac, E., Melard, A., Niang, M., Mille, C., Le Moal, G., Viard, J.P., and Rouzioux, C. 2013. Long-term antiretroviral therapy initiated during primary HIV-1 infection is key to achieving both low HIV reservoirs and normal T cell counts. The Journal of antimicrobial chemotherapy 68(5): 1169-1178. doi: 10.1093/jac/dks533.

Hutter, G., Nowak, D., Mossner, M., Ganepola, S., Mussig, A., Allers, K., Schneider, T., Hofmann, J., Kucherer, C., Blau, O., Blau, I.W., Hofmann, W.K., and Thiel, E. 2009. Long-term control of HIV by CCR5 Delta32/Delta32 stem-cell transplantation. The New England journal of medicine 360(7): 692-698. doi: 10.1056/NEJMoa0802905.

Iyidogan, P., and Anderson, K.S. 2014. Current perspectives on HIV-1 antiretroviral drug resistance. Viruses 6(10): 4095-4139. doi: 10.3390/v6104095.

Jain, V., Hartogensis, W., Bacchetti, P., Hunt, P.W., Hatano, H., Sinclair, E., Epling, L., Lee, T.H., Busch, M.P., McCune, J.M., Pilcher, C.D., Hecht, F.M., and Deeks, S.G. 2013. Antiretroviral therapy initiated within 6 months of HIV infection is associated with lower T-cell activation and smaller HIV reservoir size. The Journal of infectious diseases 208(8): 1202-1211. doi: 10.1093/infdis/jit311.

Jiang, G., Mendes, E.A., Kaiser, P., Wong, D.P., Tang, Y., Cai, I., Fenton, A., Melcher, G.P., Hildreth, J.E., Thompson, G.R., Wong, J.K., and Dandekar, S. 2015. Synergistic Reactivation of Latent HIV Expression by 
Ingenol-3-Angelate, PEP005, Targeted NF-kB Signaling in Combination with JQ1 Induced p-TEFb Activation. PLoS pathogens 11(7): e1005066. doi: 10.1371/journal.ppat.1005066.

Katlama, C., Deeks, S.G., Autran, B., Martinez-Picado, J., van Lunzen, J., Rouzioux, C., Miller, M., Vella, S., Schmitz, J.E., Ahlers, J., Richman, D.D., and Sekaly, R.P. 2013. Barriers to a cure for HIV: new ways to target and eradicate HIV-1 reservoirs. Lancet 381(9883): 2109-2117. doi: 10.1016/s0140-6736(13)60104-

$\mathrm{x}$.

Khalili, K., Kaminski, R., Gordon, J., Cosentino, L., and Hu, W. 2015. Genome editing strategies: potential tools for eradicating HIV-1/AIDS. Journal of neurovirology 21(3): 310-321. doi: 10.1007/s13365-0140308-9.

Kobayashi, M., Yoshinaga, T., Seki, T., Wakasa-Morimoto, C., Brown, K.W., Ferris, R., Foster, S.A., Hazen, R.J., Miki, S., Suyama-Kagitani, A., Kawauchi-Miki, S., Taishi, T., Kawasuji, T., Johns, B.A., Underwood, M.R., Garvey, E.P., Sato, A., and Fujiwara, T. 2011. In Vitro antiretroviral properties of S/GSK1349572, a next-generation HIV integrase inhibitor. Antimicrobial agents and chemotherapy 55(2): 813-821. doi: 10.1128/aac.01209-10.

Li, J.Z., and Gandhi, R.T. 2014. The sooner, the better: more evidence that early antiretroviral therapy lowers viral reservoirs in HIV-infected infants. The Journal of infectious diseases 210(10): 1519-1522. doi: 10.1093/infdis/jiu298.

Liang, J., Mesplede, T., Oliveira, M., Anstett, K., and Wainberg, M.A. 2015. The combination of the R263K and T66I resistance substitutions in HIV-1 integrase is incompatible with high level viral replication and the development of high-level drug resistance. Journal of virology. doi: 10.1128/jvi.01881-15.

Llibre, J.M., Pulido, F., Garcia, F., Garcia Deltoro, M., Blanco, J.L., and Delgado, R. 2015. Genetic barrier to resistance for dolutegravir. AIDS reviews 17(1): 56-64.

Luzuriaga, K., Tabak, B., Garber, M., Chen, Y.H., Ziemniak, C., McManus, M.M., Murray, D., Strain, M.C., Richman, D.D., Chun, T.W., Cunningham, C.K., and Persaud, D. 2014. HIV type 1 (HIV-1) proviral 
reservoirs decay continuously under sustained virologic control in HIV-1-infected children who received early treatment. The Journal of infectious diseases 210(10): 1529-1538. doi: 10.1093/infdis/jiu297.

Martin, A.R., and Siliciano, R.F. 2015. Progress Toward HIV Eradication: Case Reports, Current Efforts, and the Challenges Associated with Cure. Annual review of medicine. doi: 10.1146/annurev-med011514-023043.

Mesplede, T., Moisi, D., Oliveira, M., Ibanescu, I., Ohnona, F., Brenner, B., and Wainberg, M.A. 2015.

Dolutegravir inhibits HIV-1 Env evolution in primary human cells. AIDS (London, England) 29(6): 659-665. doi: $10.1097 /$ qad. 0000000000000606 .

Mesplede, T., Quashie, P.K., Osman, N., Han, Y., Singhroy, D.N., Lie, Y., Petropoulos, C.J., Huang, W., and Wainberg, M.A. 2013. Viral fitness cost prevents HIV-1 from evading dolutegravir drug pressure.

Retrovirology 10: 22. doi: 10.1186/1742-4690-10-22.

Mesplede, T., and Wainberg, M.A. 2014. Is Resistance to Dolutegravir Possible When This Drug Is Used in First-Line Therapy? Viruses 6(9): 3377-3385. doi: 10.3390/v6093377.

Mesplede, T., and Wainberg, M.A. 2015. Resistance against Integrase Strand Transfer Inhibitors and Relevance to HIV Persistence. Viruses 7(7): 3703-3718. doi: 10.3390/v7072790.

Mousseau, G., Kessing, C.F., Fromentin, R., Trautmann, L., Chomont, N., and Valente, S.T. 2015. The Tat Inhibitor Didehydro-Cortistatin A Prevents HIV-1 Reactivation from Latency. mBio 6(4). doi:

10.1128/mBio.00465-15.

Mylvaganam, G.H., Silvestri, G., and Amara, R.R. 2015. HIV therapeutic vaccines: moving towards a functional cure. Current opinion in immunology 35: 1-8. doi: 10.1016/j.coi.2015.05.001.

Osman, N., Mesplede, T., Quashie, P.K., Oliveira, M., Zanichelli, V., and Wainberg, M.A. 2015.

Dolutegravir maintains a durable effect against HIV replication in tissue culture even after drug washout. The Journal of antimicrobial chemotherapy 70(10): 2810-2815. doi: 10.1093/jac/dkv176. 
Pace, M., and Frater, J. 2014. A cure for HIV: is it in sight? Expert review of anti-infective therapy 12(7): 783-791. doi: 10.1586/14787210.2014.910112.

Purcell, D.F., Elliott, J.H., Ross, A.L., and Frater, J. 2013. Towards an HIV cure: science and debate from the International AIDS Society 2013 symposium. Retrovirology 10: 134. doi: 10.1186/1742-4690-10-134. Quashie, P.K., Mesplede, T., Han, Y.S., Oliveira, M., Singhroy, D.N., Fujiwara, T., Underwood, M.R., and Wainberg, M.A. 2012. Characterization of the R263K mutation in HIV-1 integrase that confers low-level resistance to the second-generation integrase strand transfer inhibitor dolutegravir. Journal of virology 86(5): 2696-2705. doi: 10.1128/jvi.06591-11.

Quashie, P.K., Mesplede, T., Han, Y.S., Veres, T., Osman, N., Hassounah, S., Sloan, R.D., Xu, H.T., and Wainberg, M.A. 2013a. Biochemical analysis of the role of G118R-linked dolutegravir drug resistance substitutions in HIV-1 integrase. Antimicrobial agents and chemotherapy 57(12): 6223-6235. doi: 10.1128/aac.01835-13.

Quashie, P.K., Mesplede, T., and Wainberg, M.A. 2013b. Evolution of HIV integrase resistance mutations. Current opinion in infectious diseases 26(1): 43-49. doi: 10.1097/QCO.0b013e32835ba81c.

Quashie, P.K., Oliviera, M., Veres, T., Osman, N., Han, Y.S., Hassounah, S., Lie, Y., Huang, W., Mesplede, T., and Wainberg, M.A. 2015. Differential Effects of the G118R, H51Y, and E138K Resistance Substitutions in Different Subtypes of HIV Integrase. Journal of virology 89(6): 3163-3175. doi: 10.1128/jvi.03353-14.

Saez-Cirion, A. 2015. HIV-1 virological remission for more than 11 years after interruption of early initiated antiretroviral therapy in a perinatally-infected child. 8th IAS Conf. on HIV Pathogenesis, Treatment and Prevention 19-22 July 2015, Vancouver: MOAA0105LB.

Saez-Cirion, A., Bacchus, C., Hocqueloux, L., Avettand-Fenoel, V., Girault, I., Lecuroux, C., Potard, V., Versmisse, P., Melard, A., Prazuck, T., Descours, B., Guergnon, J., Viard, J.P., Boufassa, F., Lambotte, O., Goujard, C., Meyer, L., Costagliola, D., Venet, A., Pancino, G., Autran, B., and Rouzioux, C. 2013. Post- 
treatment HIV-1 controllers with a long-term virological remission after the interruption of early initiated antiretroviral therapy ANRS VISCONTI Study. PLoS pathogens 9(3): e1003211. doi: 10.1371/journal.ppat.1003211.

Seki, T., Suyama-Kagitani, A., Kawauchi-Miki, S., Miki, S., Wakasa-Morimoto, C., Akihisa, E., Nakahara, K., Kobayashi, M., Underwood, M.R., Sato, A., Fujiwara, T., and Yoshinaga, T. 2015. Effects of raltegravir or elvitegravir resistance signature mutations on the barrier to dolutegravir resistance in vitro.

Antimicrobial agents and chemotherapy 59(5): 2596-2606. doi: 10.1128/aac.04844-14.

Siliciano, J.D., and Siliciano, R.F. 2014. Recent developments in the search for a cure for HIV-1 infection: targeting the latent reservoir for HIV-1. The Journal of allergy and clinical immunology 134(1): 12-19. doi: 10.1016/j.jaci.2014.05.026.

Simonetti, F.R., and Kearney, M.F. 2015. Review: Influence of ART on HIV genetics. Current opinion in HIV and AIDS 10(1): 49-54. doi: 10.1097/coh.0000000000000120.

Solomon, D.A., and Sax, P.E. 2015. Current state and limitations of daily oral therapy for treatment. Current opinion in HIV and AIDS 10(4): 219-225. doi: 10.1097/coh.0000000000000165.

Thornhill, J., Fidler, S., and Frater, J. 2015. Advancing the HIV cure agenda: the next 5 years. Current opinion in infectious diseases 28(1): 1-9. doi: 10.1097/qco.0000000000000123.

Wainberg, M., Anstett, K., Mesplede, T., Quashie, P., Han, Y., and Oliveira, M. 2014. The R263K mutation in HIV integrase that is selected by dolutegravir may actually prevent clinically relevant resistance to this compound. Journal of the International AIDS Society 17(4 Suppl 3): 19518. doi: 10.7448/ias.17.4.19518. Wainberg, M.A., and Han, Y.S. 2015. Will drug resistance against dolutegravir in initial therapy ever occur? Frontiers in pharmacology 6: 90. doi: 10.3389/fphar.2015.00090.

Wares, M., Mesplede, T., Quashie, P.K., Osman, N., Han, Y., and Wainberg, M.A. 2014. The M50I polymorphic substitution in association with the R263K mutation in HIV-1 subtype B integrase increases 
drug resistance but does not restore viral replicative fitness. Retrovirology 11: 7. doi: 10.1186/17424690-11-7.

White, K.L., Raffi, F., and Miller, M.D. 2014. Resistance analyses of integrase strand transfer inhibitors within phase 3 clinical trials of treatment-naive patients. Viruses 6(7): 2858-2879. doi:

10.3390/v6072858.

Whitney, J.B., Hill, A.L., Sanisetty, S., Penaloza-MacMaster, P., Liu, J., Shetty, M., Parenteau, L., Cabral, C., Shields, J., Blackmore, S., Smith, J.Y., Brinkman, A.L., Peter, L.E., Mathew, S.I., Smith, K.M., Borducchi, E.N., Rosenbloom, D.I., Lewis, M.G., Hattersley, J., Li, B., Hesselgesser, J., Geleziunas, R., Robb, M.L., Kim, J.H., Michael, N.L., and Barouch, D.H. 2014. Rapid seeding of the viral reservoir prior to SIV viraemia in rhesus monkeys. Nature 512(7512): 74-77. doi: 10.1038/nature13594. 
Table 1. Major resistance pathways to RAL, EVG and DTG

\begin{tabular}{|c|c|c|c|c|}
\hline & Mutational pathways & & resist: & \\
\hline & & RAL & EVG & DTG \\
\hline Y143 pathway & Y143C & $<10$ & $<2$ & $<2$ \\
\hline & Y143R & $<50$ & $<2$ & $<2$ \\
\hline & T97A/Y143C & $>100$ & $<2$ & $<2$ \\
\hline & T97A/Y143R & $>100$ & $<2$ & $<2$ \\
\hline & L74M/T97A/Y143G & $<50$ & ND & $<2$ \\
\hline & L74M/T97A/E138A/Y143C & $<20$ & ND & $<2$ \\
\hline N155 pathway & $\mathrm{N} 155 \mathrm{~N}$ & $<50$ &, 50 & $<2$ \\
\hline & E92Q/N155H & $<100$ & $>100$ & $<10$ \\
\hline & L74M/N155H & $<50$ & $<50$ & $<2$ \\
\hline Q148 pathway & Q148H & $<20$ & $<10$ & $<2$ \\
\hline & Q148K & $<100$ & $<100$ & $<2$ \\
\hline & Q148R & $<50$ & $<100$ & $<2$ \\
\hline & E138K/Q148H & $<10$ & $<20$ & $<2$ \\
\hline & E138K/Q148K & $>100$ & $>100$ & $<10$ \\
\hline & E138K/Q148R & $>100$ & $>100$ & $<10$ \\
\hline & G140S/Q148H & $>100$ & $>100$ & $<20$ \\
\hline & G140S/Q148K & $<10$ & $<100$ & $<2$ \\
\hline
\end{tabular}




\begin{tabular}{|l|c|c|c|c|}
\hline & G140S/Q148R & $>100$ & $>100$ & $<10$ \\
& E138A/G140S/Y143H/Q148H & $>100$ & ND & $<50$ \\
\hline R263K & R263K & $<1$ & 3 & 4 \\
pathway & R263K/H51Y & $3-5$ & 3 & $4-6$ \\
pathway & G118R & $10-17$ & $>5$ & $>8$ \\
& G118R/H51Y & ND & ND & ND \\
& G118R/E138K & $4-20$ & $4-5$ & $8-13$ \\
\hline ND: not detected.
\end{tabular}




\section{Figures:}

Figure 1. Chemical structures of (a) RAL, (b) EVG, and (c) DTG and their binding modes to the prototype foamy virus (PFV) integrase active site.

Figure 2. Hypothesized effects of DTG on a functional HIV cure.

Figure 1:
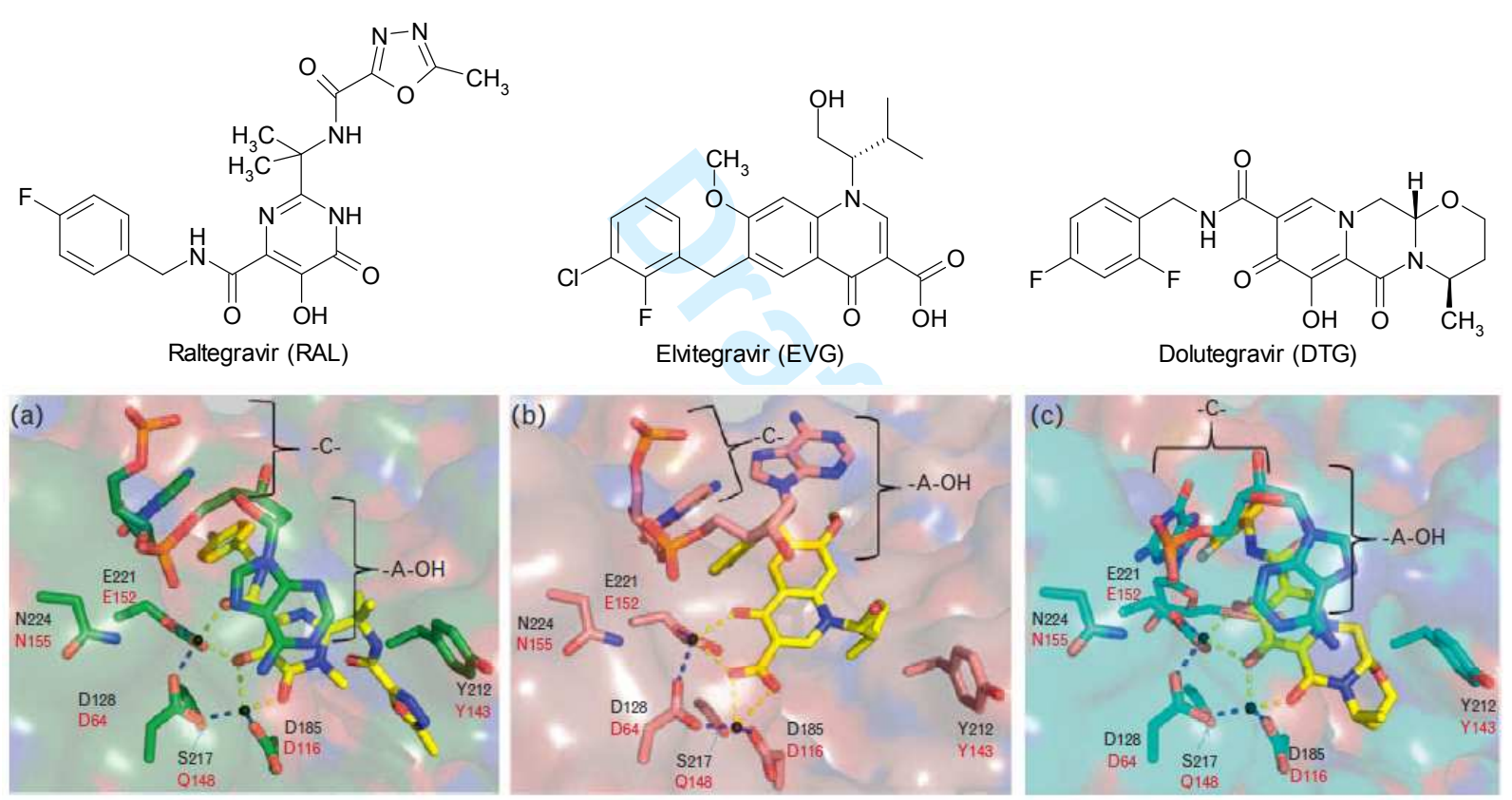
Figure 2:

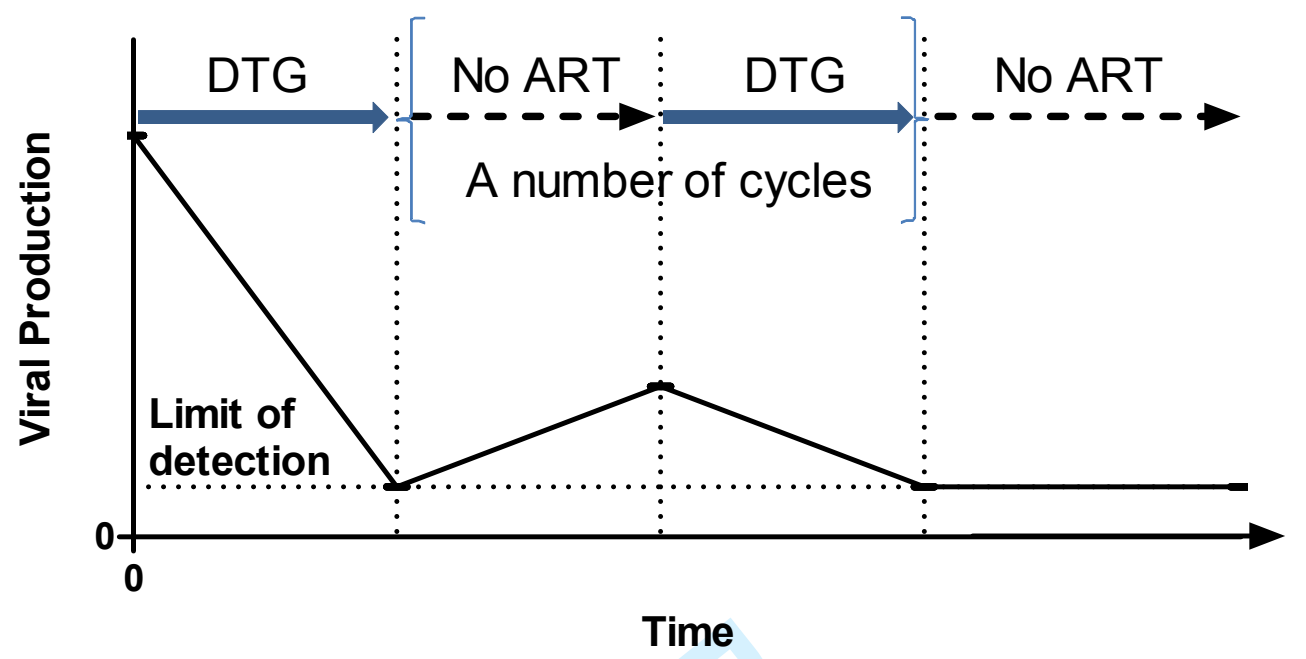


Table 1. Major resistance pathways to RAL, EVG and DTG

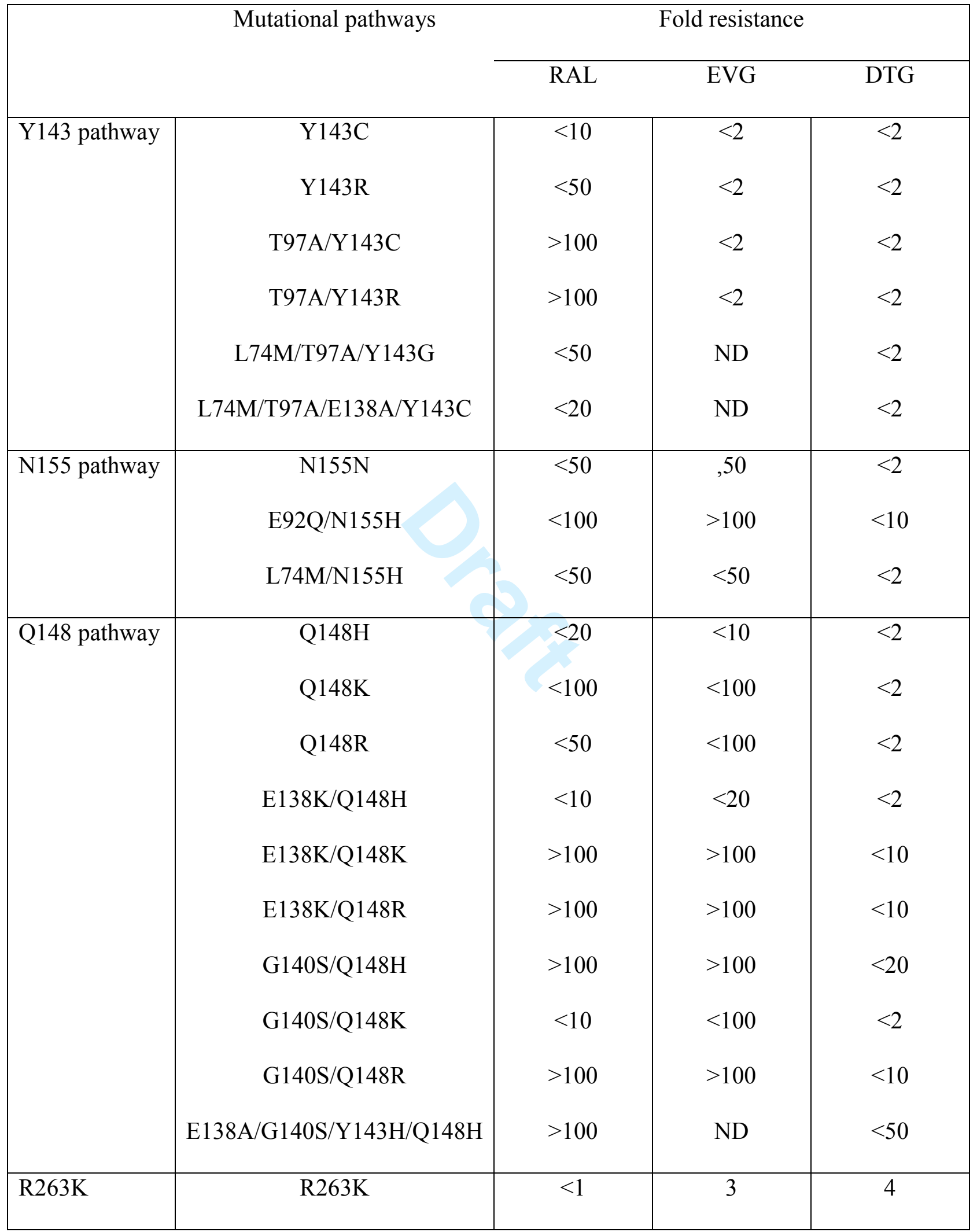




\begin{tabular}{|l|c|c|c|c|}
\hline pathway & R263K/H51Y & $3-5$ & 3 & $4-6$ \\
\hline G118R & G118R & $10-17$ & $>5$ & $>8$ \\
& G118R/H51Y & ND & ND & ND \\
& G118R/E138K & $4-20$ & $4-5$ & $8-13$ \\
\hline ND: not detected. & & & \\
\end{tabular}


Figure 1:

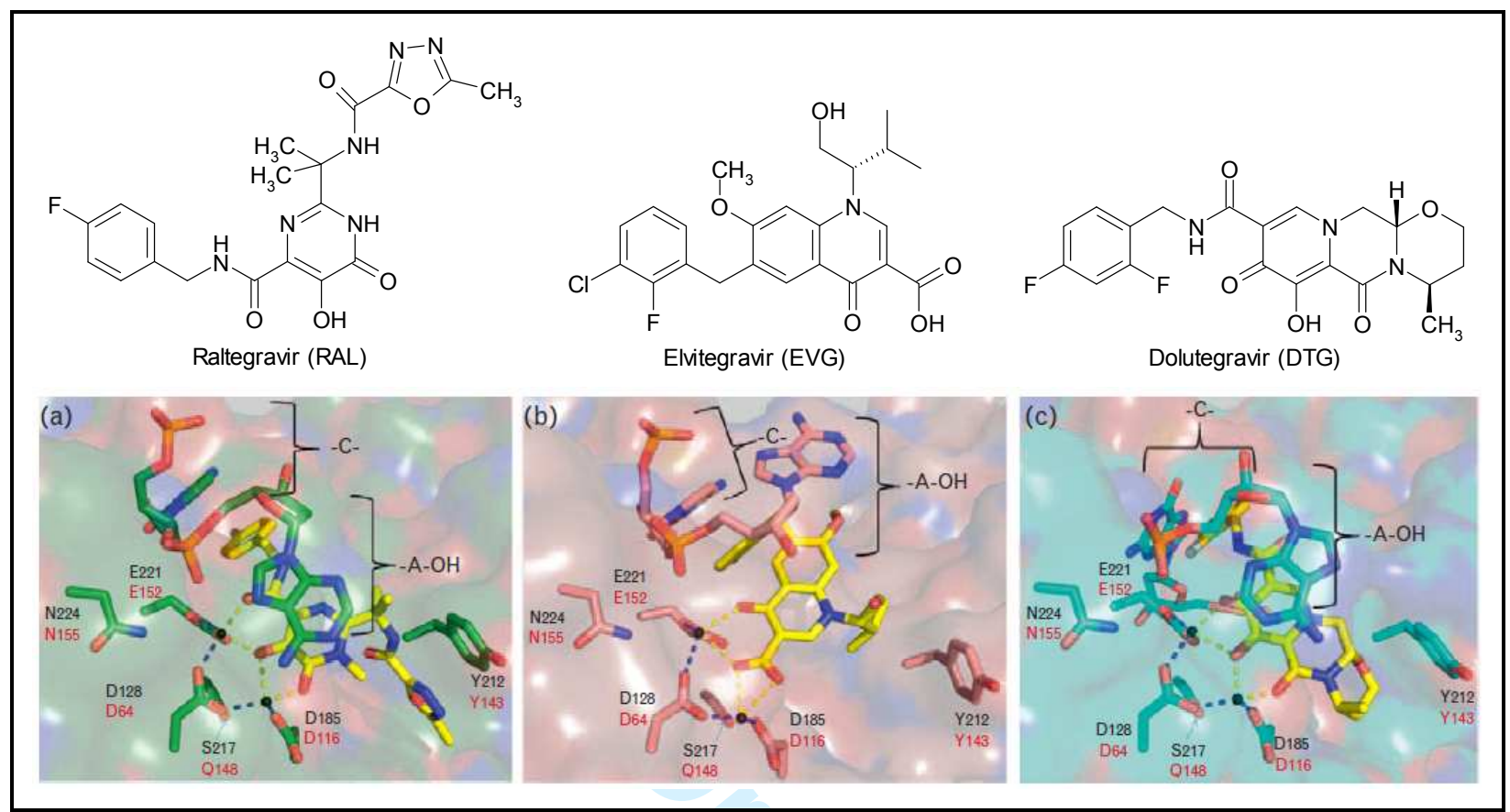


Figure 2:

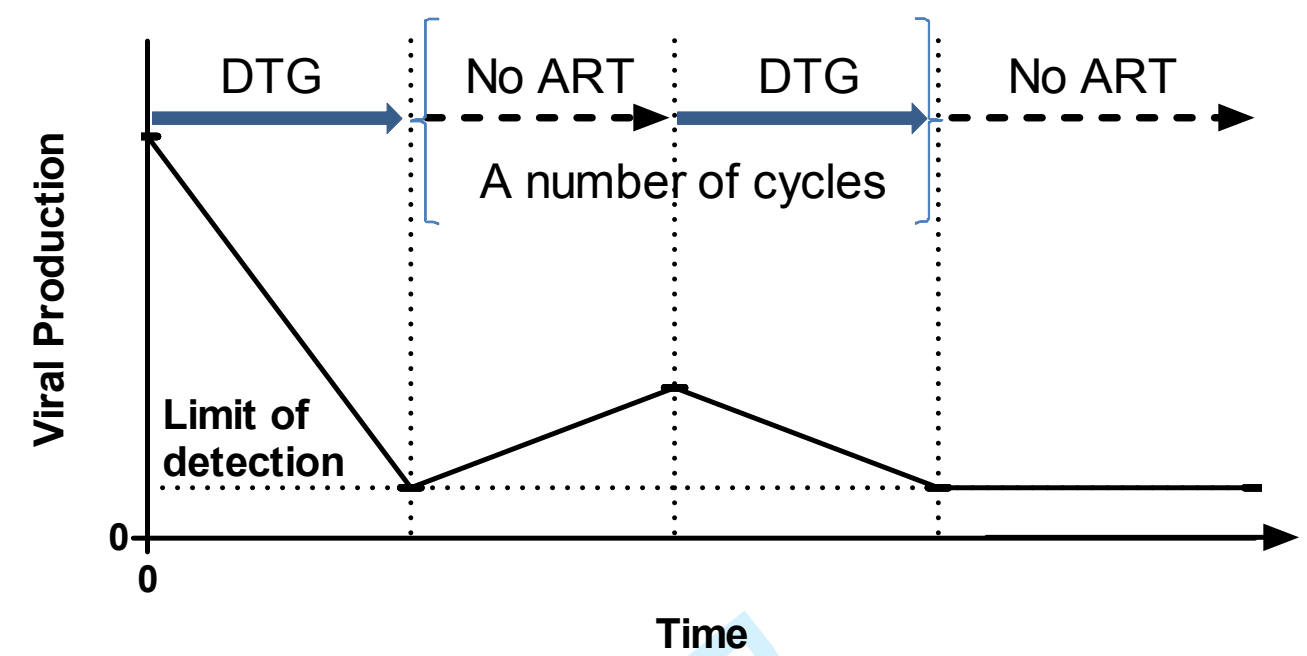

